\title{
Spinal Mechanisms of Pain Control
}

\author{
Geoffrey T. Desmoulin, BSc (Hons), MSc, Nasif I. Yasin, MD, BCh, FRCP (C), \\ and Darryl W. Chen, $B S c, D D S$
}

\begin{abstract}
Objectives: To demonstrate initial results using Khan Kinetic Treatment (KKT) as a chronic neck pain treatment and to present pain control mechanistic theory on which the treatment is based.
\end{abstract}

Methods: A self-reported functional assessment, neck pain questionnaire, and pain medication dose were used as outcome measures for 44 matched patients randomly split into 2 groups ("treatment" and "control"). The treatment group underwent a treatment period consisting of several individual KKT treatments, whereas the control group continued conventional therapy.

Results: Compared with a control group, the treatment group lowered both their self-recorded neck pain scores $(P=0.012)$ and pain medication dose $(P=0.048)$, although current functional assessment questionnaires (range of motion, overall activity, and recreation/work activities) did not detect changes $(P=0.233,0.311$, and 0.472 , respectively).

Discussion: We address the theory of the pain control mechanisms of the device in detail. Although we await randomized placebo controlled trials and additional results from ongoing mechanistic studies, initial results show that KKT is potentially an effective treatment for chronic neck pain and may contribute to the reduction of pain relieving medication.

Key Words: spine, Khan Kinetic Treatment, mechanism, pain, whiplash

(Clin J Pain 2007;23:576-585)

$\mathrm{T}_{\mathrm{D}}^{\mathrm{h}}$ he Khan Kinetic Treatment (KKT), manufactured by Datrend Systems Inc (Richmond, British Columbia, Canada), is a medical device for the treatment of spinerelated abnormalities causing pain. Combining methodologies from Physical Medicine and Rehabilitation, Neurophysiology, and Clinical Biomechanics, the KKT uses high-frequency small-amplitude sinusoidal waves to vibrate the vertebrae and repeatedly activate associated neuromuscular structures, which evoke multiple mechanisms of pain relief (see Discussion). The KKT is currently

Received for publication February 2, 2007; revised April 29, 2007; accepted May 6, 2007.

From the Wayne State University, Detroit, MI.

Reprints: Geoffrey T. Desmoulin, BSc (Hons), MSc, Optima Health Solutions International Corporation, 308-828 West 8th Avenue, Vancouver, BC, Canada V5Z 1E2 (e-mail: geoffd@wayne.edu).

Copyright $(0) 2007$ by Lippincott Williams \& Wilkins being used and further developed by Optima Health Solutions International Corporation (Optima). Device design, research, development, and manufacturing operations conform to the International Organization for Standardization standard 13485:2003 (No. 9309). KKT has class 2 approvals by the Medical Devices Bureau of Health Canada (No. 68884) and a 510 (k) from the Center for Devices and Radiological Health of the Food and Drug Administration (No. K060043). The KKT is also being operated in select hospital settings within Germany and China. The results summarized here are a consolidation of data recently acquired by using the device in a clinical setting on patients with chronic (longer than $6 \mathrm{mo}$ ) neck pain. ${ }^{1}$ Hence, this manuscript is a prelude to additional placebo/sham-controlled clinical outcome studies and compliments our on-going mechanistic research.

\section{SCOPE OF THE PROBLEM}

The overall frequency of troublesome neck pain is estimated to be approximately $34 \%$ and although the reporting rate of females is greater than that of males, approximately $14 \%$ of a randomly selected population meets the criteria for chronic neck pain $(>6 \mathrm{mo}){ }^{1}$ Whiplash, a specific form of neck pain resulting from acceleration-deceleration injury, typically associated with a motor vehicle accident, resulted in incidences ranging from 14.5 per 1000 American working women ${ }^{2}$ to 0.1 per 1000 of the New Zealand general population. ${ }^{3}$ One could reason then that the 29 billion dollars spent on whiplash diagnosis, treatment, insurance, and litigation in the United States could be scaled accordingly. ${ }^{4}$ In Canada, 18 million was spent on whiplash reimbursement and compensation alone, ${ }^{5}$ with the greatest financial burden coming from a small group of persons developing chronic symptoms. ${ }^{5}$ Lack of effective association between cause (mechanism) and effect (symptoms) are the primary causes of these numbers and their associated costs. ${ }^{6}$ The need to develop evidence-based treatment approaches to minimize the impact of neck pain in society is required.

\section{BASIS OF KKT TREATMENT}

KKT treatment is best described by comparing known mechanisms of pain relief with a novel spinal injury model proposed by a group at the Yale University School of Medicine based on spine stability (Fig. 1). ${ }^{7}$ Panjabi ${ }^{8}$ identified 3 subsystems contributing to spine stability: (1) the passive subsystem consisting of the vertebrae and facet joints, ligaments, and intervertebral 


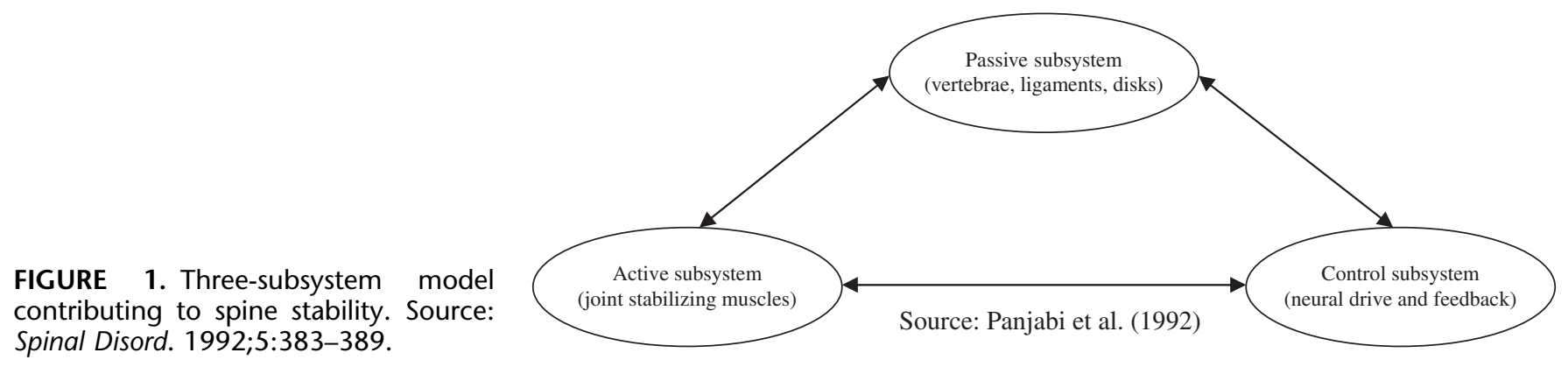

disks; (2) the active subsystem consisting of the muscles and tendons surrounding the spinal column; (3) the neural and feedback subsystem consisting mainly of the control centers that excite and coordinate the active subsystem. A dysfunction in any one of these 3 subsystems can contribute to instability of the spine and, therefore, lead to subsequent injury and pain.

Although, the Discussion section covers KKT mechanisms of pain relief in detail, we believe that KKT acts on the Yale injury model in the following ways: (1) passive subsystem by stimulating biosynthesis of intervertebral disks and replacing abnormal instantaneous axis of rotation of intervertebral joints; (2) neural and control subsystem by activating spinal cord circuitry that "gates" pain transmission and reducing gamma motor neuron activity, which reduces both involuntary muscular activation and enhanced reflex activity; and (3) active subsystem by relaxing paraspinal muscles ensuring asymmetrical loads on the spine are minimized and decreasing pain increases muscle coordination which plays a critical role in spine stabilization to prevent further injury.

\section{OBJECTIVES AND HYPOTHESES}

The objective of the study was to show initial results demonstrating that the KKT has the potential of being an effective chronic neck pain treatment option. Our hypothesis was that if the combined mechanisms of pain relief of the KKT are more effective than conventional treatment alone, then the majority of the treatment group would display improvement after the posttreatment period on a self-reported functional assessment, a neck pain questionnaire, and pain medication dose when compared with a control group.

The basis for this hypothesis was formulated from the results of numerous case study reviews when using the device as a treatment of neck pain in our clinic.

\section{METHODS}

\section{Ethics}

Ethics approval was obtained from the Institutional Review Board Services, for the procedures outlined in this paper. The procedures were also conducted according to the Helsinki Declaration ${ }^{9}$ and clearly explained to all participants before participation. Participants were asked to sign a consent form agreeing to full participation in the research protocol. They were also informed that they could withdraw from the study at any time without repercussions. All information regarding the participants has been kept confidential.

\section{Experimental Design}

This was an initial results study that examined the ability of the treatment to cause changes in patient outcome, both in self-reported levels of neck pain, overall mobility in activities of daily living and pain medication dose. The design was a control-matched, randomly assigned clinical outcome study. All participants who signed the consent form were first matched to the best of our ability regarding sex, age, and diagnosis. We randomly split the matched pairs into 2 groups: treatment and control. Although all participants eventually underwent KKT treatment, the treatment group underwent the treatment period as soon as possible after signing the consent form. The control group continued with their current conventional treatment until the treatment match finished the treatment period. At that time, both the control match and treatment participant underwent data acquisition for a second time.

\section{Participants}

A total of 44 participants, 24 female and 20 male, were recruited. These participants were between the ages of 18 and 70 [43 (14)y; mean (SD)] and had recurrent history of varying levels of chronic neck pain. A summary of the diagnoses, mechanism of injury, and current medications are shown in Tables 1 to 3 .

\section{Summary of KKT and Protocol}

The KKT is a spinal and upper cervical treatment device consisting of a controller mounted on top of an impulse delivery mechanism, or device head, which is mounted on a movable armature to a fixed stand (Fig. 2). The device head generates waveforms and the stylus located at the base of the device head mechanically transduces the waveforms through the skin and ultimately to the spine, causing minor vibration of the vertebrae and minor repetitive stretching/activation of the attached soft tissues. The device head may be freely moved in 3 dimensions so that the stylus may be positioned accurately on the skin. The stylus invokes multiple pain relieving mechanisms by delivering sinusoidal waveforms 


\section{TABLE 1. Diagnosis Summary for 44 Patients}

\begin{tabular}{lc}
\hline Diagnosis & No. Patients (\%) \\
\hline DJD* with associated ligament strain & $11(25)$ \\
Cervical disk herniation with associated ligament & $10(23)$ \\
$\quad$ strain & $5(11)$ \\
Cervical disk bulge or herniation only & $4(9)$ \\
Ligament strain only (C3-C6) with muscular & $3(7)$ \\
$\quad$ symptoms & $3(7)$ \\
Cervical DJD and osteoarthritis & $3(7)$ \\
Scoliosis & $2(5)$ \\
DJD and osteoarthritis & $2(5)$ \\
Healed vertebrae fractures & $1(2)$ \\
Cervical disk herniation, DJD, and osteoarthritis & \\
DJD with associated ligament strain and cervical & \\
$\quad$ herniation &
\end{tabular}

*DJD indicates degenerative joint disease.

of various frequencies (80 to $120 \mathrm{~Hz}$ ) and amplitudes (maximum displacement $=5 \mathrm{~mm}$ ) both linearly and rotationally to the spinous or transverse processes of the spine through the skin the stylus invokes multiple pain relieving mechanisms (see Discussion). As the device head is fixed in location, a collapsible rod provides a necessary element of safety to the patient. The rod has been designed to collapse under sufficient force that indicates a nonclinical incident (ie, the patient moves out of position). The position of the rod is being tracked by a Hall effect sensor. Thus, if the rod collapses, the device turns off within a few milliseconds. In addition, before treatment, the patient receives a thumb depressed "halt" switch which, when depressed, immediately stops the device from continuing treatment.

At patient intake, 3 digital radiographs are taken of the cervical spine (coronal, sagittal, and transverse planes). Software receiving the patients' intake digital radiographs (Spinalytics) automatically calculates the 3-dimensinal orientation ( $x, y$, and $z$ ) of the atlas as compared with the occipital condyles $(\mathrm{C} 0)$ and the axis $(\mathrm{C} 2)$.

Clinical decisions for the treatment follow standard clinical protocol. Hence, although the angles calculated by Spinalytics determine the orientation of the stylus ( 180 degrees to pathologic orientation), physical examination, diagnosis, current medications, and clinical experience dictate the overall treatment plan. Although we reserve the right to apply the device anywhere along the spine, we

\begin{tabular}{lc}
\hline TABLE 2. Mechanism of Injury Summary for 44 Patients \\
\hline Mechanism of Injury & No. Patients (\%) \\
\hline Motor vehicle accident & $14(32)$ \\
Sports collisions & $9(20)$ \\
Falls & $7(16)$ \\
Insidious (degenerative over time) & $6(14)$ \\
Repetitive strain injury & $3(7)$ \\
Trauma (unspecified) & $2(5)$ \\
Unknown & $2(5)$ \\
Fibromyalgia & $1(2)$ \\
\hline
\end{tabular}

TABLE 3. Summary of Current Pain Medication for 44 Patients

\begin{tabular}{lc}
\hline Medication & No. Patients (\%) \\
\hline NSAIDs & $10(23)$ \\
As above in combination with muscle relaxants & $15(34)$ \\
As above in combination with anti-depressants & $10(23)$ \\
Not currently taking pain medication & $5(11)$ \\
Opioids & $2(5)$ \\
Only muscle relaxants & $1(2)$ \\
Only antidepressants & $1(2)$ \\
\hline
\end{tabular}

have found the best clinical results are achieved at most spinal levels when the waveforms are applied to the transverse process of the atlas, as we did in this study (Fig. 2). Once the precise location and type (amplitude, frequency, duration of pulses, and number of pulses) of necessary treatment is determined, the "treatment" parameters are saved for a particular patient and either sent to the KKT from a desktop computer or directly programmed into the KKT using its touch-screen software located on the device head. Either way, the data are electronically archived for each patient.

After appropriately placing the patient on the treatment table, the device head is manipulated in 3 dimensions. To ensure the appropriate impulse vector, the controller has accelerometers to obtain precise stylus position feedback on the touch-screen display. When the correct impulse vector of the stylus is achieved, the device head is lowered so that the stylus makes skin contact and is then locked in position before treatment. Only then the device may begin when initiated by the clinician. This approach removes all forms of human error from the active treatment protocol.

For the treatment group, the treatment period consisted of 6 to 12 individual KKT treatments lasting 6 to 12 minutes conducted over a period of 3 to 6 weeks. The control group continued with current conventional treatment.

\section{KKT Treatment Flow Diagram}

As per the International Organization for Standardization standard 13485:2003 for Quality Management of medical devices, the KKT protocol has been standardized. The flow diagram in Figure 3 outlines the approved treatment protocol and is the flow of treatment any patient undergoing treatment would receive.

\section{Data Acquisition}

The self-assessed neck pain questionnaire used in this study was consistent with the standard format of the Pain Outcomes Questionnaire developed by the American Academy of Pain Management. ${ }^{10}$ Both the treatment and matched control patients described their neck pain on an ordinal scale ranging from 0 to 10 at intake of initial assessment before the treatment period began. At the end of the treatment period for the treatment patients, each matched patient (treatment and control) again recorded the self-assessed neck pain score. 

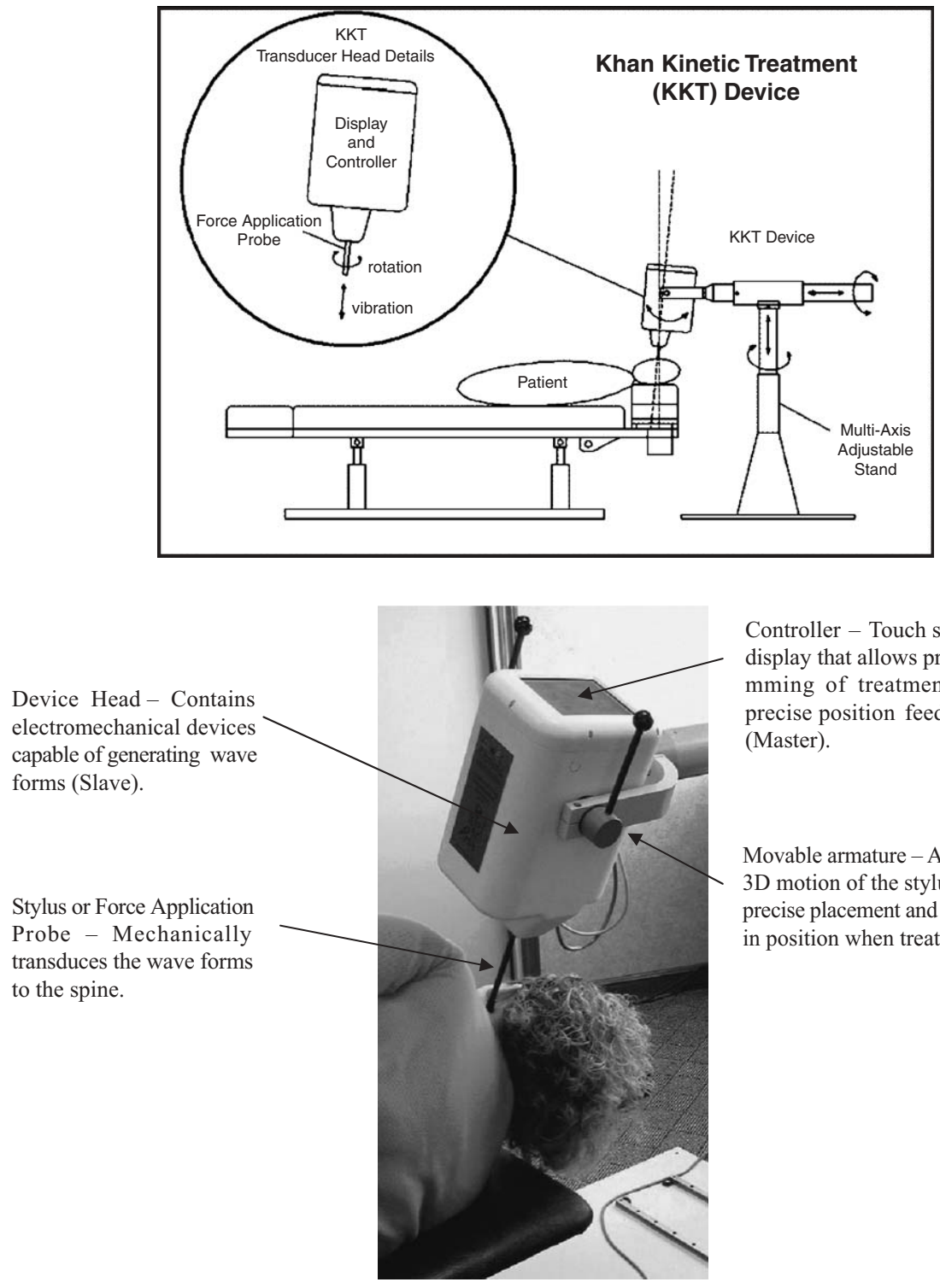

Controller - Touch screen display that allows programming of treatment and precise position feedback (Master).

Movable armature - Allows 3D motion of the stylus for precise placement and locks in position when treating.

FIGURE 2. KKT treatment set-up.

Three functional self-assessment measures consisted of (1) a general mobility or range of motion score (0 to $100)$, (2) an overall activity level score (0 to 100), and (3) a recreation/work activities level score (0 to 100). These functional assessments were recorded for both group's pretreatment and posttreatment period of the treatment group. Scoring 100 on any of these tests meant that, despite the self-assessed neck pain score, the patients have full function in the areas indicated, and a score of 0 meant that the self-assessed neck pain has incapacitated the patient to complete bed rest. It is noted that these are unconventional self-assessments. However, they were already in use by the clinic in which participants were recruited. Hence, early results could be obtained quickly, because current patients could be recruited as participants for this trial.
Medication types were recorded and grouped into several categories: analgesics and nonsteroidal antiinflammatory drugs (NSAIDs), anticonvulsants (muscle relaxants), opioids, and antidepressants. The doses were noted by the patient pretreatment and posttreatment period for both groups and collectively grouped into 2 categories: same or decreased.

\section{Data Analysis}

Descriptive statistics (frequencies, means, and standard deviations) have been reported so that comparisons of similar data throughout the literature can be made. As a reliability and validity study of implementing ordinal scales in the assessment of self-reported pain has already been established, ${ }^{10}$ we will not address these issues here. A 2 -way $t$ test was performed to determine whether or not 


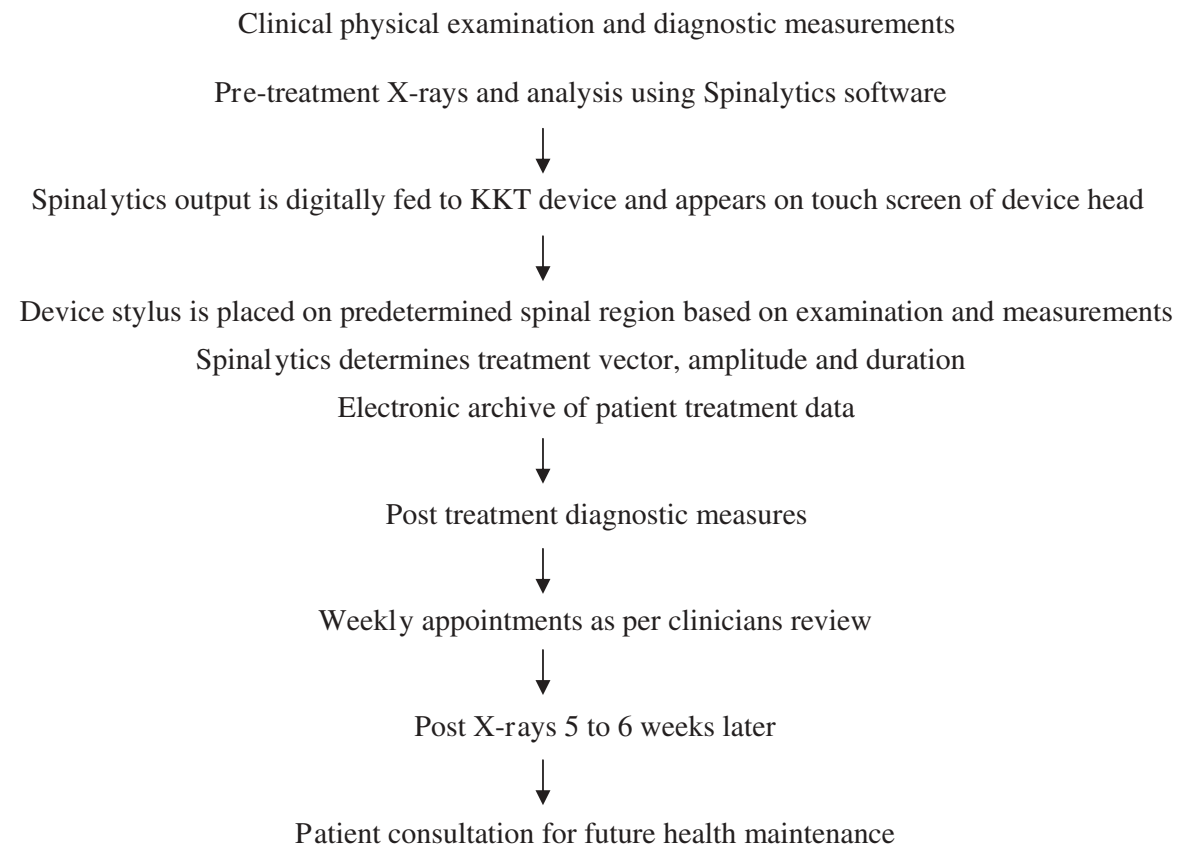

FIGURE 3. Quality approach to treatment methodology.

the severity of the initial self-reported neck pain scores [initial neck pain (iNP)] differed between the treatment and control groups before study commencement. A 2-group (treatment vs. control) by 2-neck pain outcome (positive and neutral) $\chi^{2}$ test was used to analyze the self-reported neck pain scores before and after (diffNP) the KKT treatment period.

Paired $t$ tests were used to analyze the 3 functional activity measures and to show that pretreatment measures did not differ between the groups. Although data ranging from 0 to $10 / 100$ could be considered ordinal data, the $t$ test robustness in distributional violations is considered a valid test in this case (Dr Robert W. Schutz, personnel communication, December 1, 2005).

A 2-group (treatment and control) by 2-pain medication dose outcome (same, decreased) $\chi^{2}$ test was used to analyze the reported changes in medication doses. Also, a 2-group (treatment and control) by 3-pain medication types (NSAIDs, NSAIDs plus muscle relaxants, and NSAIDs, muscle relaxants, and antidepressants) $\chi^{2}$ test was used to analyze the differences in medication types between groups.

Using G-Power software, ${ }^{11}$ post hoc power was calculated for all outcome measures: (1) 2-group (treatment vs. control) by 2-neck pain outcome (diffNP) (neutral and positive) $\chi^{2}$ test (Power $=0.512$ ), (2) 2-group (treatment vs. control) by range of motion assessment $t$ test (Power $=0.495$ ), (3) 2-group (treatment vs. control) by activity level $t$ test (Power $=0.495)$, (4) 2-group (treatment vs. control) by recreation/work activity level $t$ test (Power $=0.367$ ), and (5) 2-group (treatment vs. control) by 2-pain medication dose outcome (same, decreased) $\chi^{2}$ test $($ Power $=0.437)$.

\section{RESULTS}

\section{Neck Pain Outcome}

Figure 4 shows the frequency distribution and quantile box plot of the iNP scores from all patients (iNP). A paired $t$ test determined that the severity of the self-reported neck pain scores before KKT treatment did not differ between the treatment and control groups $(P=0.889)$ (Fig. 5).

Figure 6 shows the means, standard error, and standard deviation for all patients $(\mathrm{N}=44)$ in the 2 groups after the treatment period. Table 4 shows the JMP software $2 \times 2$ output table that was used to conduct the test. The treatment group had significantly lower selfreported neck pain scores posttreatment period (diffNP) when compared with control patients $(P=0.012)$.

\section{Functional Assessment Outcome}

Self-reported functional assessment scores were also compared. Three paired $t$ tests were performed to see whether or not the groups differed from one another before the initiation of treatment. The tests show that none of the functional assessment scores (range of motion; overall activity, and a recreation/work activities) of either group were significantly different before treatment $(P=0.320,0.241$, and 0.665 , respectively). Repeating these tests, posttreatment period showed that none of the functional assessment scores (range of motion, overall activity, and a recreation/work activities) were significantly different when compared with the same measurements in the control group $(P=0.233,0.311$, and 0.472 , respectively). 


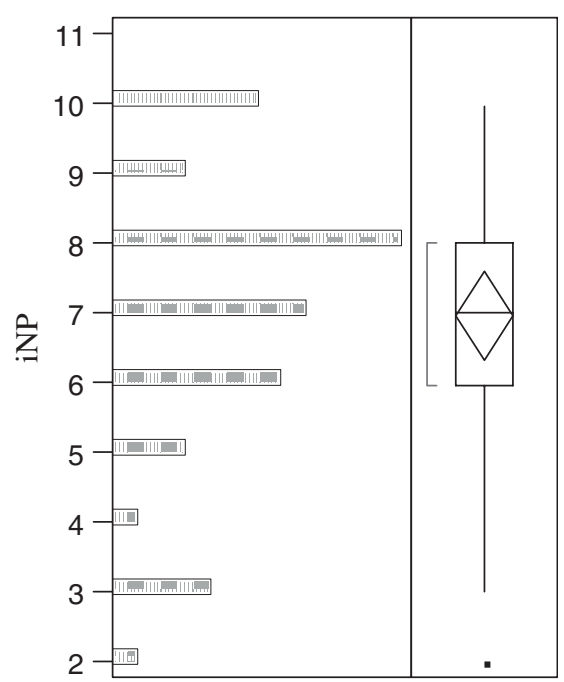

FIGURE 4. Quality box and frequency distribution for the iNP scores for all patients $(\mathrm{N}=44)$.

\section{Pain Medication Dose Outcome}

Eighty-nine percent of patients were taking some form of medication to treat their neck pain. However, a 2-group (treatment and control) by 2-pain medication (yes or no) $\chi^{2}$ test showed that neither group was significantly different from each other $(P=0.635)$. As well, there was no significant difference between the groups and the types of medication (NSAIDs, NSAIDs combined with muscle relaxants, and NSAIDs combined both with muscle relaxants and antidepressants) they were taking to treat their neck pain $(P=0.207)(\mathrm{N}=35)$, as only 2 patients were taking opioids (1 was taking muscle relaxants, 1 was taking antidepressants) and 5 patients were not taking any pain medications. However, a 2-group (treatment by control) by 2-pain medication dose outcome (same or reduced) showed that the treatment group had significantly reduced the dose of their medications (no matter what type) when compared with the control group $(P=0.048)$ (Table 5$)$. $\mathrm{N}$ for this test was reduced to 36 patients in total, 18 from each group. Five patients ( 3 control and 2 treatment) were currently not on any medication to treat their neck pain. Hence, these patients (5) along with their matched patients (3) were removed from the $\chi^{2}$ test ( 8 in total).

\section{DISCUSSION}

\section{KKT Effectiveness}

The preliminary results of the self-recorded neck pain scores suggested that when the current prototype of KKT is used as outlined in the Methods section, it is a potentially effective treatment alternative for people with neck pain of multiple etiologies. Although we hold promise in these introductory results, we cannot differentiate between those patients truly helped by the device

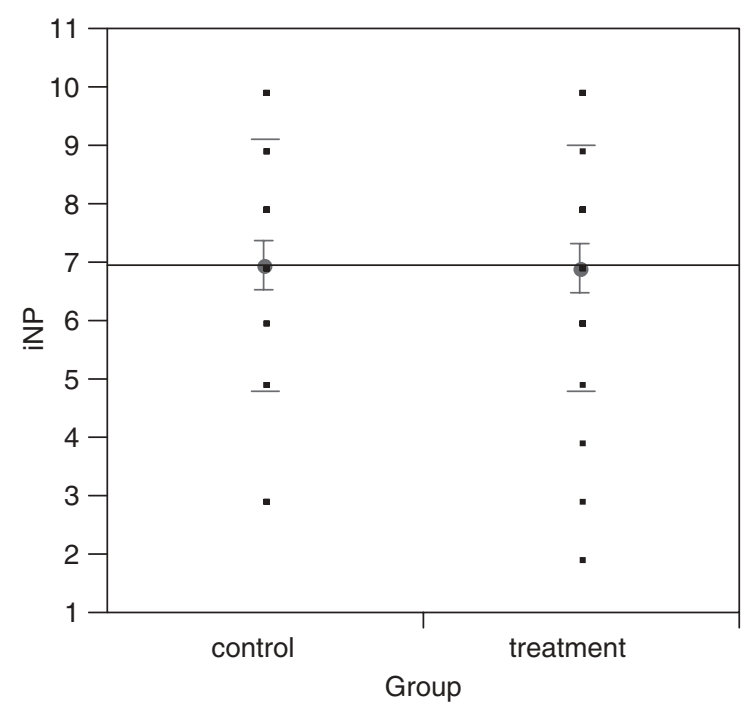

FIGURE 5. Mean (dot), \pm 1 standard error (solid bar), \pm 1 standard deviation (broken bar) of scatter plot data from iNP scores from the 2 groups ( $N=44 ; 22$ patients in each group).

and those experiencing a placebo effect. Hence, we require a large placebo/sham-controlled trial to fully explain these early findings.

The results of the 3 self-reported functional assessment questions (range of motion, overall activity, and recreation/work activities) did not significantly differ when compared with the control group. These types of questions were chosen for their ease of data collection, ease of patient understanding, and the fact that they were

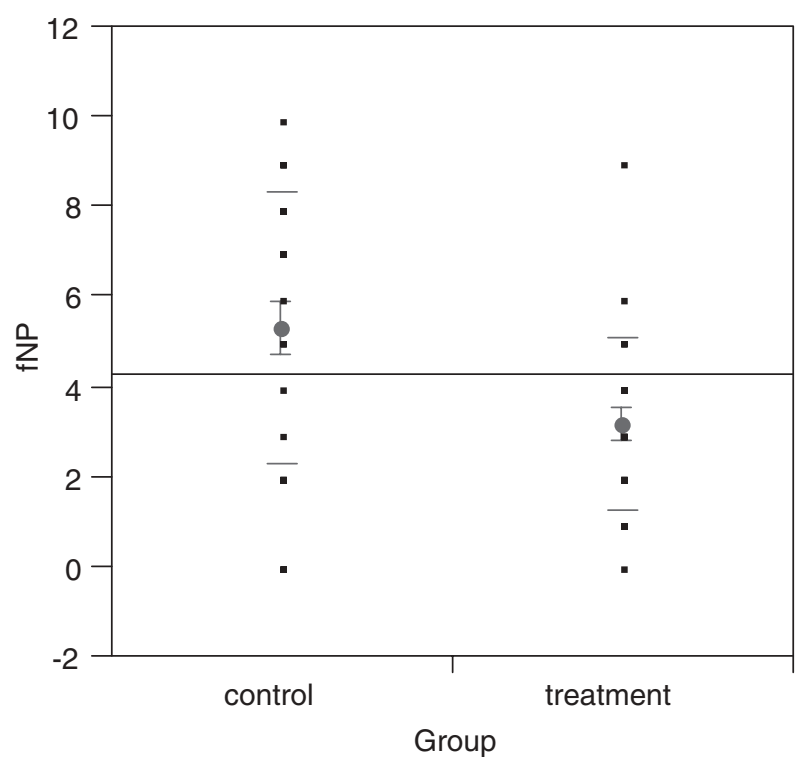

FIGURE 6. Mean (dot), \pm 1 standard error (solid bar), \pm 1 standard deviation (broken bar) of scatter plot data from posttreatment period ( $\mathrm{fNP}$ ) scores from the 2 groups $(\mathrm{N}=44$; 22 patients in each group). 
TABLE 4. Two-group (Treatment and Control) by 2-diffNP Outcome (Neutral or Positive) Table Used for $\chi^{2}$ Analysis

\begin{tabular}{lccc}
\hline diffNP & Group & \multirow{2}{\text{Treatment}}{} & \\
\cline { 2 - 3 } Count & Control $(\mathbf{N}=\mathbf{2 2})$ & & $\mathbf{2 2})$ \\
\hline Positive & 10 & 18 & 28 \\
Neutral & 12 & 4 & 16 \\
& 22 & 22 & 44 \\
\hline
\end{tabular}

$\mathrm{N}=44 ; 22$ patients in each group.

already in use by the clinic who recruited patients for the study. This allowed for recruitment of patients who had already recently undergone an intake examination. Unfortunately, the format of these questions does not follow any standard seen in literature. This may be a contributing factor as to why we did not detect changes in these measurements. However, they were already in use by the clinic in which the patients were recruited. Hence, results could be obtained quickly since current patients could be recruited as participants for this trial. Future studies will include a more detailed evaluation of range of motion, vitality, and impairments when completing activities of daily living using current standardized questionnaires in reference to KKT treatment. Initial results demonstrate that the treatment group reduced their dosage of pain medications posttreatment period when compared with a control group. Although these initial results will require further explanation with higher quality placebo/sham-controlled studies, results of the combination of both decreased levels of perceived neck pain and decreases in pain medication have been encouraging, despite differences in neck pain etiology.

\section{Effect of Vertebrae Linear Displacement}

Traditionally, over the centuries, spinal manipulation or impulse (low-amplitude high-velocity) treatment has been performed using bare hands. ${ }^{12}$ As the number of investigations using this methodology increases, it is becoming evident that the variability of patient outcomes implementing this type of treatment can be significant. ${ }^{12,13}$ These results may be because of the variability in the pressure applied by the hand owing to variations in practitioner hand anatomy, ${ }^{14}$ variability in patient anatomy, ${ }^{15,16}$ or the variability of the application itself. ${ }^{17}$

TABLE 5. Two-group (Treatment and Control) by 2medication Dose Outcome (Same or Decreased) Table Used for $\chi^{2}$ Analysis

\begin{tabular}{lccc}
\hline Dose & Group & Treatment & \\
\cline { 2 - 3 } Count & Control $(\mathbf{N}=\mathbf{1 8})$ & & $(\mathbf{N}=\mathbf{1 8})$ \\
\hline Reduced & 2 & 8 & 10 \\
Same & 16 & 10 & 26 \\
& 18 & 18 & 36 \\
\hline
\end{tabular}

$\mathrm{N}=36 ; 18$ patients in each group.
In fact, despite the many published randomized clinical trials (RCT), a substantial number of reviews and several national clinical guidelines, controversy remains regarding the evidence for or against efficacy of spinal manipulation and mobilization for spine related pain. ${ }^{12,18,19}$

However, as we will see in the section below, there are highly specific clinical situations in which mobilization or stabilization is recommended. As the force impulse (force $\times$ time characteristic) delivered to the spine relates highly to vertebral motion and associated reflex activity, ${ }^{20}$ it is an important aspect of spine manipulation to be able to precisely control as KKT does. ${ }^{14}$

\section{Hypo-hyper mobile Joints}

Many manual therapy approaches recommend mobilization or manipulation interventions if patients lack spine mobility and present with no sign of contraindications. ${ }^{21-23}$ Hence, the treatment of choice for hypomobile joints of the spine causing pain is therapeutic manipulation, which causes spine segment mobility. ${ }^{24}$ In the past, determining whether a patient has hypomobile spinal joints has been unreliable. ${ }^{25-27}$ However, more recent research clumping the outcomes to 1 of 2 or 3 choices (hypomobile, normal, and hypermobile) in combination with other tests increased interrater reliability significantly. ${ }^{28-30}$ In fact, the outcome of a recent well-conducted RCT shows that patients judged to have hypomobile spinal joints responded better to manipulation than those without hypomobile joints. ${ }^{24}$ As well, failure rates for hypomobile patients practicing stabilization and conditioning exercises as the sole treatment method was $74 \%$ compared with only $26 \%$ of those same candidates who received only spinal manipulation as the mode of treatment.

In contrast, hypermobile joints may be best dealt with using exercises specific to conditioning muscles that act to stabilize those same joints. ${ }^{31,32}$ Indeed, the outcome of the same RCT as above ${ }^{24}$ shows that patients judged to have hypermobile spinal joints had improved more than people treated with the same stabilization and conditioning exercises that did not present with hypermobile joints. Further, the treatment failure rates climbed to $83 \%$ when using spinal manipulation on hypermobile patients and only $22 \%$ when conducting exercise specific for conditioning muscles that increase joint stability. Part of this exercise program included low impact aerobic conditioning.

Although, several researchers have found that spinal manipulation of the vertebrae is a useful treatment for spine related pain, ${ }^{33,34}$ it is apparent that one type of treatment cannot apply to all cases all of the time. ${ }^{35}$ Hence, we theorize that the success of the KKT for both hyper and hypomobile joints works by replacing abnormal cervical instantaneous axes of rotation in hypermobile joints and help mobilize hypomobile joints to normal ranges. We are currently collecting instantaneous axes of rotation data to help explain this portion of the devices success. 


\section{Spinal Effects of Specific Frequency Waveforms Gamma Motor Neuron Modulation}

As a result of induced muscle pain, muscle firing patterns or coordination between flexors and extensors change significantly to reduce motion of the segment. ${ }^{36} \mathrm{It}$ has also been shown that gamma motor neuron sensitivity increases during induced muscle pain. ${ }^{37}$ Although this increase in sensitivity may not lead to excessive electromyography at all contraction levels, it most certainly increases reflexive activity. This enhanced sensitivity may act to create load asymmetries on the spine.

As the vertebrae are moved during the treatment, they stretch the muscles attached to them. As has been found previously in animal models, we note that the vibratory aspect results in the application of sinusoidal stretches to the tendons of the muscles attached to the vertebrae, resulting in decreases to gamma motor neuron input mediated by Renshaw cells activated during vibration. ${ }^{38-41}$ These researchers discovered that the inhibition increased as vibration frequency increased. The frequencies tested ranged from 100 to $300 \mathrm{~Hz}$. As well, Pompeiano et $\mathrm{al}^{40}$ discovered that Renshaw cell activity maximized at frequencies between 150 and $250 \mathrm{~Hz}$. Although, micrometer displacements are typical for these types of experiments, Pompeiano et $\mathrm{al}^{40}$ used amplitudes ranging from $180 \mu \mathrm{m}$ to $12 \mathrm{~mm}$ none of which showed a difference in Renshaw cell activity, hence the phenomenon is frequency dependent and displacement independent. ${ }^{40}$ As gamma motor neuron input decreases, so does the stretch reflex input for contraction. It has been shown that this reflex activity entering the medial branch of the dorsal ramus at one spinal level causes similar activity across 1 or 2 adjacent levels. ${ }^{42}$ Hence, not only does the application of the treatment translate as a wave down the spine at multiple spinal levels, but the reflex activity involved in that translation also acts at multiple spinal levels. So, if the paraspinal muscles are undergoing a pain-spasm-pain cycle ${ }^{43}$ or have enhanced gamma motor neuron sensitivity and are responsible for asymmetric loads on the spine, then the KKT will reduce the load asymmetry. ${ }^{44}$

\section{Cellular Biosynthesis}

There is some evidence to show that vibration affects biosynthesis of chondrocytes. ${ }^{45,46}$ Liu et al ${ }^{46}$ using a sinusoidal waveform of $1.4 \mathrm{~g}$ acceleration at 200 to $1600 \mathrm{~Hz}$ found that at 200 and $300 \mathrm{~Hz}$ the mechanical vibration of chondrocytic culture promoted DNA and proteoglycan synthesis, although frequencies above $400 \mathrm{~Hz}$ suppressed it. As well, Kasra et $\mathrm{al}^{45}$ discovered that collagen and protein synthesis of annulus fibrosus (outer layer of intervertebral disk) cells was promoted when $3 \mathrm{MPa}$ loads were delivered at the higher frequencies $(\sim 20 \mathrm{~Hz})$ tested.

One might argue that any vibration could cause these changes in the spine. However, it has been shown that exposure to frequencies close to the natural frequency of the torso-spine system $(<12 \mathrm{~Hz})$, such as those seen with truck and tractor drivers causes early onset of disk degeneration. ${ }^{24,47-49}$

The range of frequencies between 20 and $300 \mathrm{~Hz}$ caused disk biosynthesis and frequencies, below $12 \mathrm{~Hz}$ caused disk degeneration, whereas frequencies above $400 \mathrm{~Hz}$ suppressed the biosynthesis. Hence, the frequency of the KKT stylus oscillation is likely stimulating both chondrocyte and annulus fibrosus cellular constituencies to a level that may decrease disk degeneration.

Dr Christopher Hunter (http://www.eng.ucalgary.ca/ resrch_mech/Mech_Hunter.htm) from the University of Calgary has signed a long-term research collaboration agreement with Optima Health Solutions International Corporation. Together, we have completed an experiment protocol that comprises experiments covering 3 important areas of KKT treatment:

- Mechanics imparted on the disk,

- Influence on cell metabolism in the disk, and

- Optimization of the imparted mechanics.

\section{Central Mechanisms}

Specific frequency mechanical vibration applied transcutaneously reduces chronic pain. ${ }^{50,51}$ Although the mechanism is not truly understood, ${ }^{52}$ vibration analgesia relies at least in part on central nervous system processes rather than local mechanisms. ${ }^{53,54}$ It is believed that the input transduced by the mechanoreceptors in the skin interrupts central nervous system processing of the pain signal. ${ }^{53}$ This reasons as several researchers have discovered that lower frequency vibration does not cause analgesia as well as higher frequency vibration, suggesting efficacy is stimulus specific. ${ }^{50,53}$

This information supports the Melzack and Wall ${ }^{55}$ gate-control theory of pain whereby cutaneous input from Ia afferents act to close the gate or interrupt pain signals being sent through A-delta and $\mathrm{C}$ fibers, to be perceived in the cortex. Even though, the gate-control theory must be continually revised to accord with new information, it has been a major impetus for stimulating fruitful research and none the less useful in attempting to explain vibration analgesia. ${ }^{56,57}$

This mechanism is important because it is believed to act on the circuitry of the spinal cord where we have learned in a previous review article, permanent plastic changes can occur. ${ }^{58}$

\section{CONCLUSIONS}

When compared with a control group, initial results show that KKT caused significant decreases in neck pain of neuromusculoskeletal origin and decreased pain medication use but no changes in functional measures were found. Limitations of this study include not having sham controls or double blinding the patients/clinician to experimental groups. Hence, we propose that subsequent work consist of a 3 group (treatment, asymptomatic, and sham) randomized sham-controlled, double-blind, repeated measures design using more standardized and objective approaches 
to patient outcomes. The sham treatment would consist of using the device at decreased amplitude on the soft tissues of the trapezius muscle rather than on a landmark of the transverse process of the atlas.

\section{ACKNOWLEDGMENTS}

The authors thank Dr Aslam H. Khan for all his effort with assisting in data collection and competent KKT treatment of the patients and Optima Health Solutions International Corporation for its continued support for this project as a means to obtain third party research results.

\section{REFERENCES}

1. Dvorak J. Epidemiology, physical examination, and neurodiagnostics. Spine. 1998;23:2663-2673.

2. Schutt C, Dohan F. Neck injury to women in auto accidents. A metropolitan plague. JAMA. 1968;206:2689-2692.

3. Mills $\mathrm{H}$, Horne G. Whiplash-manmade disease? $N \mathrm{Z}$ Med $\mathrm{J}$. 1986;99:373-374.

4. Young W. The enigma of whiplash injury: current management strategies and controversies. Postgrad Med. 2001;109:179-180.

5. Spitzer W, Skovron M, Salmi L, et al. Scientific monograph of the Quebec Task Force on Whiplash-associated Disorders: redefining "whiplash" and its management. Spine. 1995;20(suppl):1s-73s.

6. Lonnberg F. Whiplash. Epidemiology, diagnosis and treatment. Ugeskr Laeger. 2001;163:2231-2236.

7. Bogduk N. Neck Pain. Aust Fam Physician. 1984;13:26-30.

8. Panjabi MM. The stabilizing system of the spine. Part I. Function, dysfunction, adaptation, and enhancement. J Spinal Disord. 1992;5: 383-389.

9. World Medical Organization. Declaration of Helsinki. Br Med J. 1996;313:1448-1449.

10. Clark M, Gironda R, Young R. Development and validation of the Pain Outcomes Questionnaire-VA. J Rehabil Res Dev. 2003;40: 381-396.

11. Faul F, Erdfelder E. GPOWER: A priori, post-hoc and compromise power analysis for MS-DOS (computer program). Bonn, FRG: Bonn University, Department of Psychology; 1992.

12. Assendelft W, Morton S, Yu E, et al. Spinal manipulative therapy for low back pain. A meta-analysis of effectiveness relative to other therapies. Ann Intern Med. 2003;138:871-881.

13. Haigh R, Clarke AK. Effectiveness of rehabilitation for spinal pain. Clin Rehabil. 1999;13:63-81.

14. Perle SM, Kawchuk GN. Pressures generated during spinal manipulation and their association with hand anatomy. $J$ Manipulative Physiol Ther. 2005;28:e1-e7.

15. van Roy P, Barbaix E, Clarijs JP, et al. Anatomical background of low back pain: variability and degeneration of the lumbar spinal canal and intervertebral disk. Schmerz. 2001;15:418-424.

16. Rucco V, Basadonna PT, Gasparini D. Anatomy of the iliolumbar ligament: a review of its anatomy and a magnetic resonance study. Am J Phys Med Rehabil. 1996;75:451-455.

17. Kawchuk GN, Herzog W. Biomechanical characterization (fingerprinting) of five novel methods of cervical spine manipulation. J Manipulative Physiol Ther. 1993;16:573-577.

18. Bronfort G, Haas M, Evans R, et al. Efficacy of spinal manipulation and mobilization for low back pain and neck pain: a systematic review and best evidence synthesis. Spine J. 2004;4:335-356.

19. Koes BW, Assendelft W, van der Heijden GJ, et al. Spinal manipulation for low back pain. An updated systematic review of randomized clinical trials. Spine. 1986;21:2860-2871.

20. Colloca C, Keller T, Harrison D, et al. Spinal manipulation force and duration affect vertebral movement and neuromuscular responses. Clin Biomech (Bristol, Avon). 2006;21:254-262.

21. Maitland G. Vertebral manipulation. 5th ed. Oxford: Butterworth Heinemann; 1986:74-76.

22. Jull G. Examination of the articular system. In: Boyling JD, Palastanga N, eds. Grieve's Modern Manual Therapy. Edinburgh: Churchill Livingstone; 1996:511-527.
23. Grieve G. Common vertebral joint problems. Edinburgh: Churchill Livingstone; 1989:350-368.

24. Fritz J, Whitman J, Childs J. Lumbar spine segmental mobility assessment: an examination of validity for determining intervention strategies in patients with low back pain. Arch Phys Med Rehabil. 2005;86:1745-1752.

25. Maher C, Adams R. Reliability of pain and stiffness assessments in clinical manual lumbar spine examination. Phys Ther. 1994;74: 801-809. Discussion 809-811.

26. Binkley J, Statford P, Gill C. Inter-rater reliability of lumbar accessory motion mobility testing. Phys Ther. 1995;75:786-795.

27. Phillips D, Twomey L. A comparison of manual diagnosis with a diagnosis established by a uni-level lumbar spinal block procedure. Man Ther. 1996;2:82-87.

28. Hicks G, Fritz J, Delitto A, et al. Preliminary development of a clinical prediction rule for determining which patients with low back pain will respond to a stabilization exercise program. Arch Phys Med Rehabil. 2005;86:e1-e7.

29. Flynn T, Fritz J, Whitman J, et al. A clinical prediction rule for classifying patients with low back pain who demonstrate short term improvement with spinal manipulation. Spine. 2002;27: 2835-2843.

30. Fritz J, Piva S, Childs J. Accuracy of the clinical examination to predict radiographic instability of the lumbar spine. Eur Spine $J$. 2005:14:743-750.

31. Greive G. Lumbar instability. Physiotherapy. 1982;68:2-9.

32. Paris S. Physical signs of instability. Spine. 1985;10:277-279.

33. Licciardone J, Brimhall AK, King LN. Osteopathic manipulative treatment for low back pain: a systematic review and meta-analysis of randomized controlled trials. BMC Musculoskelet Disord. 2005; 6:43.

34. Bronfort G, Haas M, Evans R, et al. Efficacy of spinal manipulation and mobilization for low back pain and neck pain: a systematic review and best evidence synthesis. Spine J. 2004;3:335-356.

35. Koes B, Assendelft W, van der Heijden G, et al. Spinal manipulation for low back pain. An updated systematic review of randomized clinical trials. Spine. 1996;21:2860-2871. Discussion 2872-2873.

36. Graven-Nielsen T, Svensson P, Arendt-Nielsen L. Effects of experimental muscle pain on muscle activity and coordination during static and dynamic motor function. Electroencephalogr Clin Neurophysiol. 1997;105:156-164.

37. Matre D, Sinkjaer T, Svensson P, et al. Experimental muscle pain increases the human stretch reflex. Pain. 1998;75:331-339.

38. Fromm C, Noth J. Reflex responses of gamma motoneurons to vibration of the muscle they innervate. J Physiol. 1976;256:117-136.

39. Fromm C, Noth J, Thilmann A. Inhibition of extensor gamma motoneurons by antagonistic primary and secondary spindle afferents. Pflugers Arch. 1976;363:81-86.

40. Pompeiano O, Wand P, Sontag K. Response of Renshaw cells to sinusoidal stretch of hind limb extensor muscles. Arch Ital Biol. 1975;113:205-237.

41. Rymer Z, Hasan Z. Prolonged time course for vibratory suppression of stretch reflex in the decebrate cat. Exp Brain Res. 1981;44: $101-112$.

42. Kang Y, Choi W, Pickar J. Electrophysiologic evidence for an intersegmental reflex pathway between lumbar paraspinal tissues. Spine. 2002;27:E56-E63.

43. Cobb C, deVries H, Urban R, et al. Electrical activity in muscle pain. Am J Phys Med. 1945;54:80-87.

44. Maigne J, Vautravers P. Mechanism of action of spinal manipulative therapy. Joint Bone Spine. 2003;70:336-341.

45. Kasra M, Goel V, Martin J, et al. Effect of dynamic hydrostatic pressure on rabbit intervertebral disc cells. J Orthop Res. 2003;21: 597-603.

46. Liu J, Sekiya I, Asai K, et al. Biosynthetic response of cultured articular chondrocytes to mechanical vibration. Res Exp Med (Berl). 2001;200:183-193.

47. Troup J. Driver's back pain and its prevention. A review of the postural, vibratory and muscular factors, together with the problem of transmitted road-shock. Appl Ergon. 1978;9:207-214. 
48. Morisi F, Raffi G, Caudarella R. Effects of low frequency vibrations on the human spine. Boll Soc Ital Biol Sper. 1979;55:190-194.

49. Bonney R, Corlett E. Vibration and spinal lengthening in simulated vehicle driving. Appl Ergon. 2003;34:195-200.

50. Ekblom A, Hansson P. Extra-segmental transcutaneous electrical nerve stimulation and mechanical vibratory stimulation as compared to placebo for the relief of acute oro-facial pain. Pain. 1985;23:223-229.

51. Guieu R, Tardy-Gervet M, Roll J. Analgesic effects of vibration and transcutaneous electrical nerve stimulation applied separately and simultaneously to patients with chronic pain. Can J Neurol Sci. 1991;18:113-119.

52. Guieu R, Tardy-Gervet M, Giraud P. Met-enkephalin and betaendorphin are not involved in the analgesic action of transcutaneous vibratory stimulation. Pain. 1992;48:83-88.
53. Roy E, Hollins M, Maixner W. Reduction of TMD pain by high frequency vibration: a spatial and temporal analysis. Pain. 2003;101:267-274. Erratum in: Pain. 104:717.

54. Tardy-Gervet M, Guieu R, Ribot-Ciscar E, et al. Transcutaneous mechanical vibrations: analgesic effect and antinociceptive mechanisms. Rev Neurol (Paris). 1993;149:177-185.

55. Melzack R, Wall P. Pain mechanisms: a new theory. Science. 1965;150:971-979.

56. Dickenson A. Gate control theory of pain stands the test of time. Br J Anaesth. 2002;88:755-757.

57. Bishop B. Pain: its physiology and rationale for management. Part III. Consequences of current concepts of pain mechanisms related to pain management. Phys Ther. 1980;60:24-37.

58. Desmoulin G, Khan A. Spinal mechanisms of chronic pain. AJPM. 2007;17:27-43. 